\title{
External Flow Analysis over Three-box form (Mercedes model) Using CFD
}

\author{
Ali Najim Abdullah \\ Ministry Of Higher Education/ Al-Rafidain University College
}

\begin{abstract}
Computational fluid dynamic software ANSYS Fluent 15.0 is used to predict the physical parameters on the external surface of a vehicle. The simulation of the wind tunnel experiments using the above CFD package would be carried out. To study the aerodynamic influence of vehicle segments are considered as threebox form passenger car model (Mercedes). As this model provided with the extremities in the design of vehicle category in summarizes these results in a broader sense to understand the aerodynamics of such profile. A wind tunnel experiments consists of physically testing the model in a simulated environment. It is a closed environment in which air is blown across the model at a constant velocity. As the air passes over the vehicle, it follows the streamlines pattern present on the body. This causes a change in pressure and velocity characteristics across the model. By studying the pressure and velocity variations across the model, the drag and lift coefficient could be predicted.
\end{abstract}

Key words: CFD, External flow field, Three-box form car, Drag coefficient, Lift coefficient.

\section{Introduction}

An accurate vehicle model a accounts are related to the accuracy in the velocity and pressure variations across the body during testing. To obtain correct results, a true representation of a vehicle is tested in a simulated wind tunnel experiment ${ }^{[1]}$. In real world, full size vehicle models are tested in a wind tunnel to obtain its behavior as shown in figure (1).

\section{Grids And Boundary Conditions}

Vehicle model is created using ANSYS Fluent 15.0. This model is an approximation to the realistic vehicle and considered for the numerical (CFD) analysis, two-box form to a study the external flow characteristic and the effect of vehicle body profile on it. The computational domain is discredited into 140197 tetrahedral elements for two-box form, as shown in figure (2). As shown in figure (3), the vehicle is placed approximately in the middle of the numerical model of the wind tunnel in the length as well as in width. There is a ground clearance in between the floor the vehicle and the bottom wall of the wind tunnel. The tunnel wall and the vehicle body are considered as typical wall boundary conditions. At the inlet of the wind tunnel, a constant mean velocity of $45 \mathrm{~m} / \mathrm{s}$ or $160 \mathrm{~km} / \mathrm{h}$ is applied and the outlet of the wind tunnel is considered as an outflow boundary. For solving the flow and turbulence parameters, a three dimensional finite volume numerical method and a standard k- $\varepsilon$ turbulence model based on commercial code ANSYS Fluent 15.0, are used for analysis.

\section{Numerical Formulation}

For solving the standard variables $\mathrm{u}, \mathrm{v}, \mathrm{w}, \mathrm{k}, \varepsilon$ and $\mathrm{T}$, three-dimensional Navier-Stokes equations for steady, turbulent, and incompressible flow ${ }^{[2]}$ can be written as:

\section{1-3-1 Governing Equations}

Mass (continuity) equation:

$$
\frac{\partial \rho}{\partial t}+\frac{\partial}{\partial x_{i}}\left(\rho u_{i}\right)=0
$$

Momentum equation:

$\frac{\partial}{\partial t}\left(\rho u_{i}\right)+\frac{\partial}{\partial x_{j}}\left(\rho u_{i} u_{j}\right)+\frac{\partial P}{\partial x_{i}}-\frac{\partial \tau_{i j}}{\partial x_{j}}=0$

Energy equation:

$\frac{\partial}{\partial t}(\rho H)+\frac{\partial}{\partial x_{i}}\left(\rho u_{i} H-u_{i} \tau_{i j}\right)=0$ 
Where the stress tensor $\tau_{i j}$,

$\tau_{i j}=\left\lceil\mu\left(\frac{\partial u_{i}}{\partial x_{j}}+\frac{\partial u_{j}}{\partial x_{i}}\right)\right\rceil-\frac{2}{3} \mu \frac{\partial u_{j}}{\partial x_{i}} \delta_{i j}$

\section{1-3-2 Turbulence modeling}

The standard k- $\varepsilon$ turbulence model ${ }^{[3]}$ is used which contain two equations one for turbulence kinetic energy and the other for its dissipation rate obtained from the following equations respectively:

$\rho \frac{\partial k}{\partial t}=\frac{\partial}{\partial x_{i}}\left\lceil\left(\mu+\frac{\mu_{t}}{\operatorname{Pr}_{k}}\right) \frac{\partial k}{\partial x_{i}}\right]+\left(2 \mu_{t} \delta_{i j}-\frac{2}{3} \rho k \delta_{i j}\right) \frac{\partial u_{j}}{\partial x_{i}}-\rho \varepsilon$

And....

$\rho \frac{\partial \varepsilon}{\partial t}=\frac{\partial}{\partial x_{i}}\left[\left(\mu+\frac{\mu_{t}}{\operatorname{Pr}_{k}}\right) \frac{\partial \varepsilon}{\partial x_{i}}\right]+C_{1 \varepsilon} \frac{\varepsilon}{k}\left(2 \mu_{t} \delta_{i j}-\frac{2}{3} \rho k \delta_{i j}\right) \frac{\partial u_{j}}{\partial x_{i}}-C_{2 \varepsilon} \rho \frac{\varepsilon^{2}}{k}$

In the above equation, the last three terms on the right hand side shows the diffusion generation, and the dissipation rates of $\varepsilon$ and the turbulent viscosity derived is,

$$
\mu_{t}=C_{\mu} \rho \frac{k^{2}}{\varepsilon}
$$

Where $C \mu=0.09$

The simulation results are obtained under the convergence criteria of $1.1^{-10}$ for the residuals of $\mathrm{u}, \mathrm{v}, \mathrm{w}, \mathrm{k}$ and $\varepsilon$. The drag coefficient can be calculated using the following equation.

$$
C_{D}=\frac{F_{D}}{\frac{1}{2} \rho_{\infty} V_{a}^{2} A_{f}}
$$

Where $F_{D}$ is the force acting in the direction of air flow on the frontal area of the vehicle body and $\left(\frac{1}{2} \rho_{a} V_{a}^{2}\right)$ is free stream dynamic pressure. In the present solution, a semi-implicit method for pressure linked equation (SIMPLE)) is used. Figure (4) shows the flow chart for external flow modeling that illustrates the options used in ANSYS Fluent 15.0 software.

\section{Results And Discussion}

Figure (5) shows the velocity increasing smoother when compared to that of the wagon. This is attributed due to the smooth profile changes across the hood and the roof. There is a subtle change in velocity at the rear end as the flow separation occurs. The pressure contour plots along $\mathrm{z}$-axis at a distance of $1 \mathrm{~m}$ from the side face( figure 6) indicates details about the low-pressure zones. This study is done to analyze the amount of down force acting on the vehicle that keeps it on the ground. The red zone of high pressure at the front face of vehicle indicates the presence of stagnation points. Air drag coefficient for Toyota model is shown in (figure 7) and the Lift coefficient for Toyota model is shown in (figure 8), such values are amended in the same study.

\section{Conclusion}

In the case of three box form model, low-pressure pockets are present at the top and bottom of the vehicle. This differential pressure is almost negligible that keeps the vehicle on ground.

\footnotetext{
Notations

A: The model frontal area $\left(\mathrm{m}^{2}\right)$.

$\mathrm{C}_{\mathrm{D}}$ : Drag coefficient.

$\mathrm{F}_{\mathrm{D}}$ : Total drag force $(\mathrm{N})$.

$\mathrm{V}_{\infty}$ : Undisturbed air flow $(\mathrm{m} / \mathrm{s})$.

$\mathrm{g}$ : Gravitational acceleration $\left(\mathrm{m} / \mathrm{s}^{2}\right)$.

$\varepsilon$ : Dissipation rate.

$\rho: \operatorname{Density}\left(\mathrm{Kg} / \mathrm{m}^{3}\right)$.

$\sigma:$ Normal stress.

$v:$ Kinematic viscosity.

$\mu_{\mathrm{t}}$ : Additional viscosity due to turbulence.

$\tau_{\mathrm{ij}}$ : Stress tensor.
} 


\section{References}

[1]. Aerodynamic Testing of road vehicles- Testing methods and procedures (Stabilized Apr 2016).

[2]. Turbulence models and their applications, T.S.D.Karthik, 10 $0^{\text {th }}$ Indo German winter Academy 2011.

[3]. Philip J. Pritchard, Fox and McDonald's, "Introduction to Fluid Mechanics", 2011.

[4]. Akshay Parab, Ammar Sakarwala, Bhushan Paste, Vaibhav Patil, Amol Mangrulkar,"Aerodynamics Analysis of a Car Model Using Fluent Ansys 14.5", International Journal On Recent Technologies On Mechanical and Electrical Engineering(IJRMEE), November 2014.

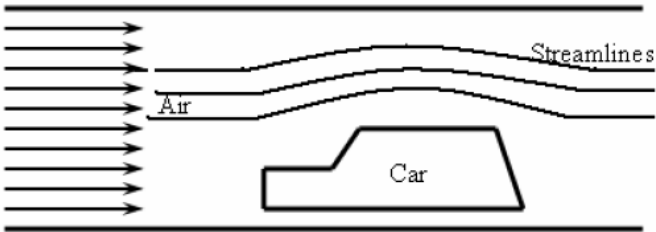

Figure(1): Wind tunnel testing

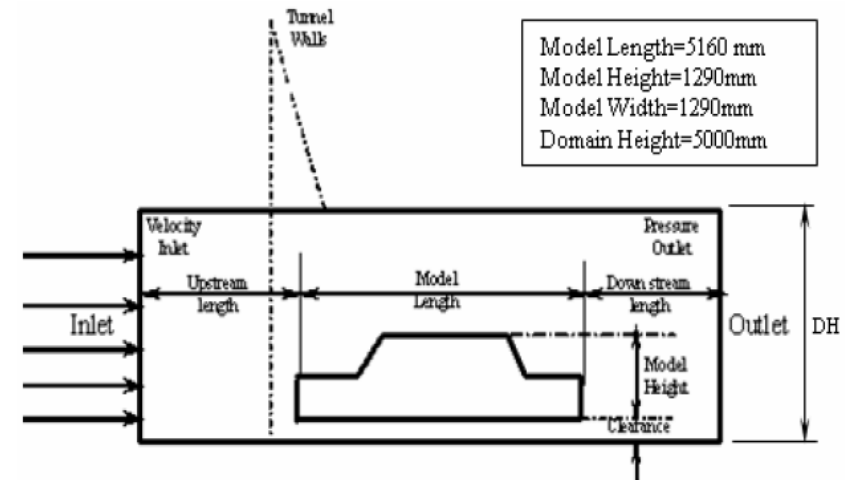

Figure(2): Grid display for Three- box form car model

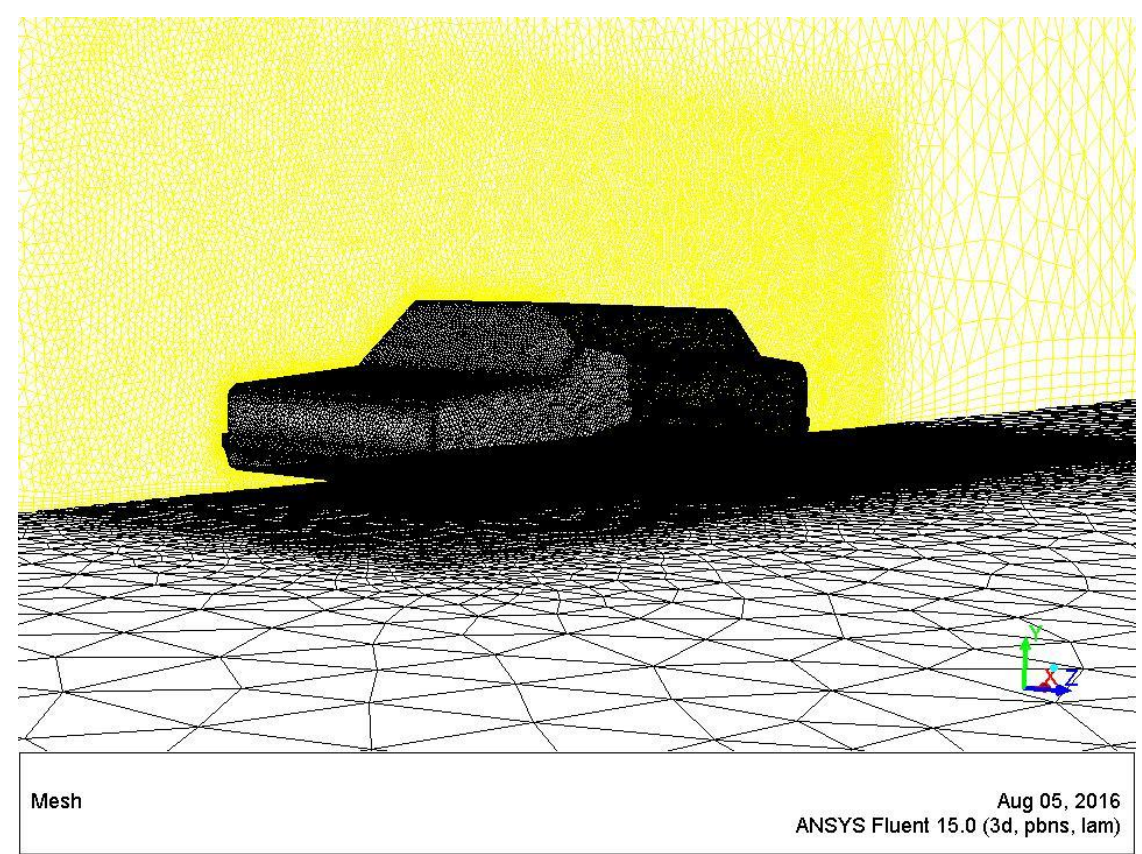

Figure(3): ANSYS Fluent 15.0 for Mercedes model 


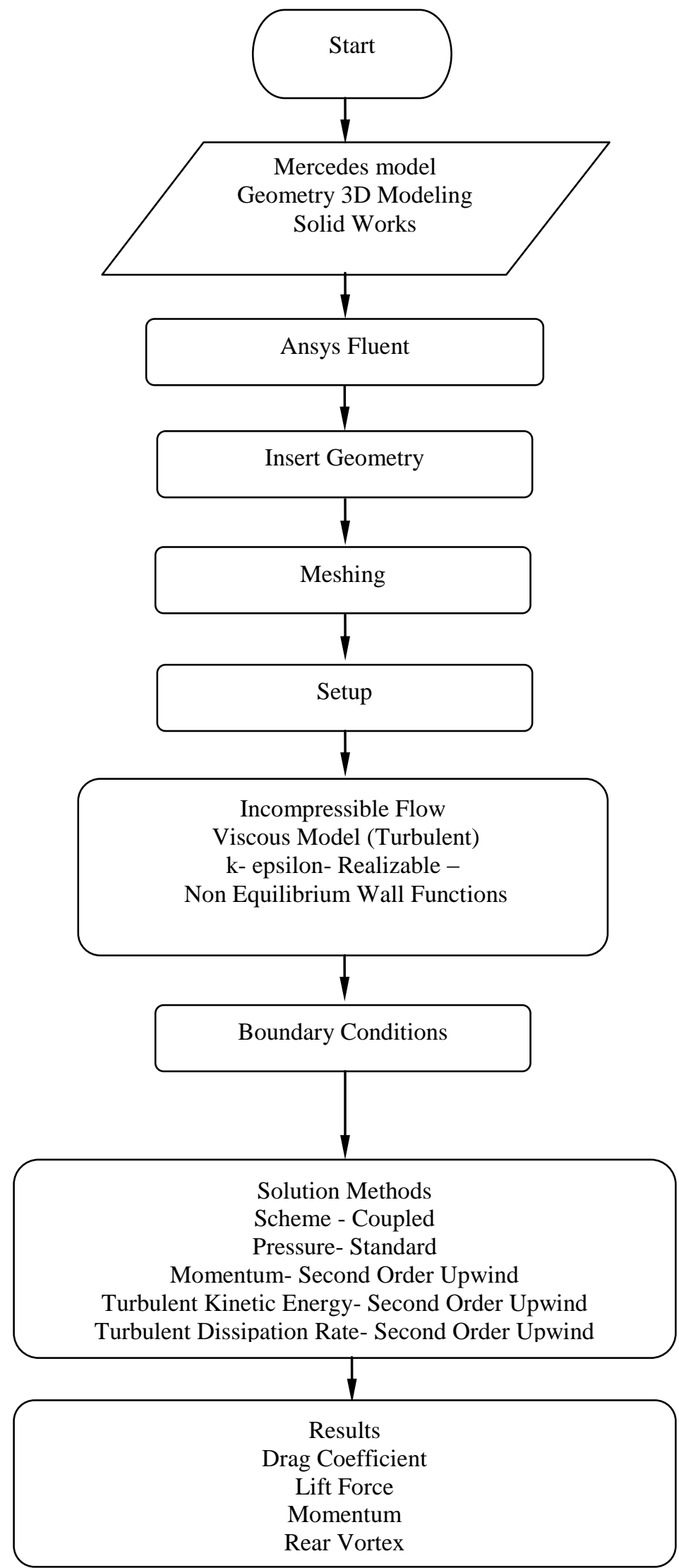

Figure (4): Shows the flow chart for the external flow modeling That illustrates the options used in ANSYS Fluent 15.0 


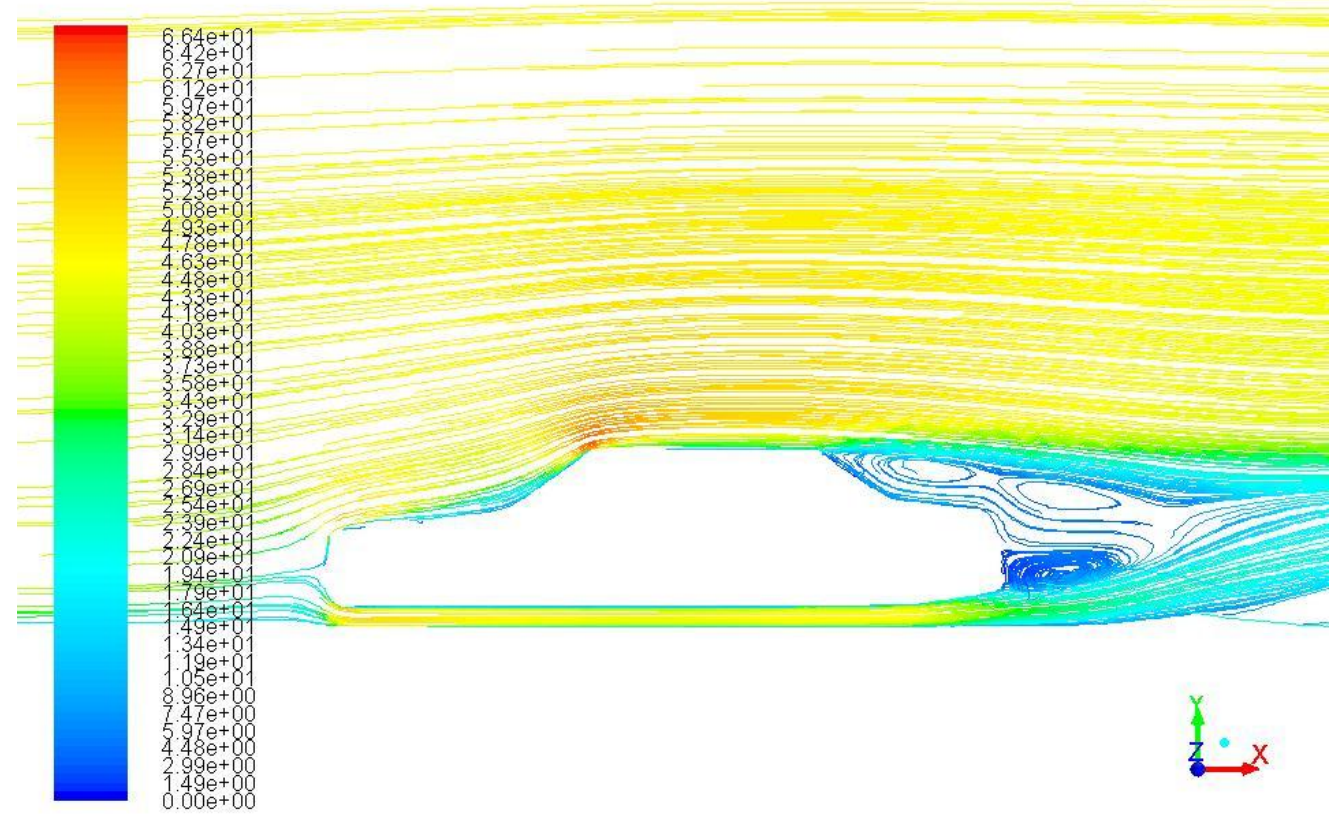

Pathlines Colored by Velocity Magnitude $(\mathrm{m} / \mathrm{s})$

Aug 07, 2016

ANSYS Fluent 15.0 (3d, pbns, rke)

Figure (5): Velosity vectors on the surface of vehicle $(\mathrm{m} / \mathrm{s})$

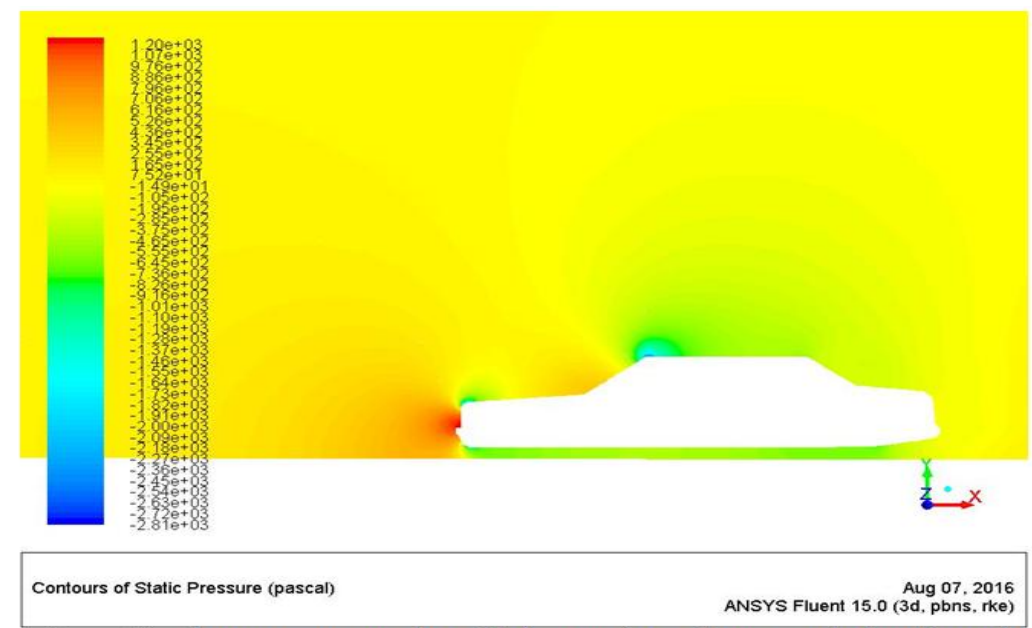

Figure(6): Pressure contour for vehicle at a plane along $z$-axis $=1 \mathrm{~m}$ (pascal)

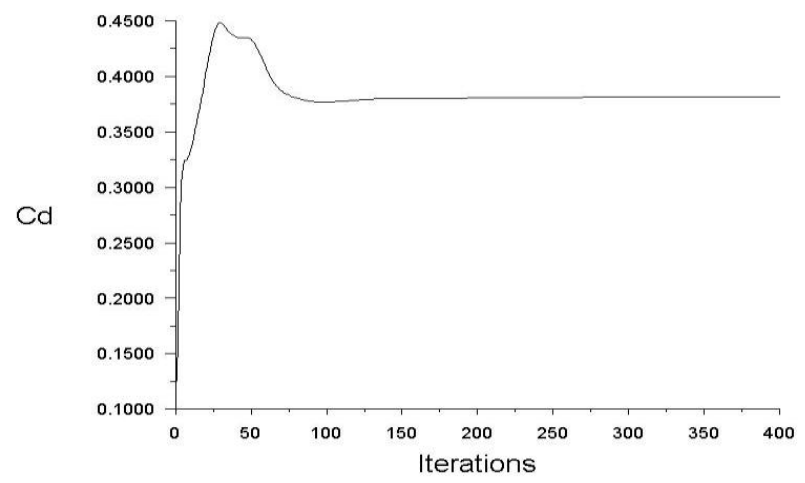

drag Convergence History

Aug 07, 2016
ANSYS Fluent 15.0 (3d, pbns, rke)

Figure (7): Air drag coefficient for Mercedes model 


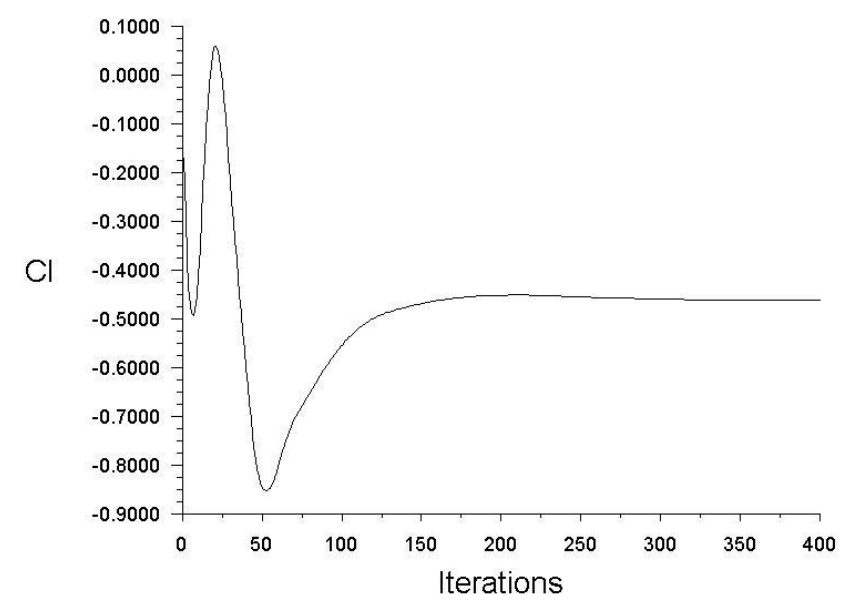

Figure (8): Lift coefficient for Mercedes model 